\title{
ORIGINAL ARTICLE \\ International Spinal Cord Injury Urinary Tract Infection Basic Data Set
}

\author{
LL Goetz ${ }^{1}$, DD Cardenas ${ }^{2}$, M Kennelly ${ }^{3}$, BS Bonne Lee ${ }^{4}$, T Linsenmeyer ${ }^{5}$, C Moser $^{6}$, J Pannek ${ }^{7}$, J-J Wyndaele ${ }^{8}$ \\ and F Biering-Sorensen ${ }^{9}$
}

Objectives: To develop an International Spinal Cord Injury (SCI) Urinary Tract Infection (UTI) Basic Data Set presenting a standardized format for the collection and reporting of a minimal amount of information on UTIs in daily practice or research.

Setting: International working group.

Methods: The draft of the Data Set developed by a working group was reviewed by the Executive Committee of the International $\mathrm{SCl}$ Standards and Data Sets, and later by the International Spinal Cord Society (ISCoS) Scientific Committee and the American Spinal Injury Association (ASIA) Board. Relevant and interested scientific and professional (international) organizations and societies ( 40) were also invited to review the data set, and it was posted on the ISCOS and ASIA websites for 3 months to allow comments and suggestions. The ISCOS Scientific Committee, Executive Committee and ASIA Board received the data set for final review and approval.

Results: The International SCI UTI Basic Data Set includes the following variables: date of data collection, length of time of sign(s)/ symptom(s), results of urine dipstick test for nitrite and leukocyte esterase, urine culture results and resistance pattern. The complete instructions for data collection and the data form itself are freely available on the website of ISCoS (http://www.iscos.org.uk).

Spinal Cord (2013) 51, 700-704; doi:10.1038/sc.2013.72; published online 30 July 2013

Keywords: spinal cord injury; spinal cord lesion; urinary tract infection; symptoms; dipstick; urine culture

\section{INTRODUCTION}

Collection of data on urinary tract infection (UTI) is universal when individuals with spinal cord lesions consult doctors with knowledge regarding spinal cord lesions. Interpretation of the data, however, is less standardized, as UTI in patients with spinal cord injury (SCI) is not unambiguously defined.

The purpose of the International SCI UTI Basic Data Set for individuals with SCI is to standardize the collection and reporting of a minimal amount of information related to a possible UTI in daily practice in accordance with the purpose and vision of the International SCI Data Sets. ${ }^{1}$ This will also make it possible to evaluate and compare the results from various published studies.

The data in the International SCI UTI Basic Data Set generally will be used in connection with data in the International SCI Core Data Set, ${ }^{2}$ which includes information on date of birth and injury, gender, the cause of spinal cord lesion and neurological status. In addition, the International SCI UTI Basic Data Set can be used in relation to the International SCI Lower Urinary Tract Function Basic Data $\mathrm{Set}^{3}$ that, among other variables, contains information on the bladder-emptying method(s), involuntary urine leakage (incontinence) and drugs for the urinary tract within the last year including antibiotics/antiseptics for treatment of UTI or for prophylactic reasons.
A spinal cord lesion may be traumatic or non-traumatic in etiology. All lesions to the spinal cord, conus medullaris and cauda equina are included in the present context.

It is extremely important that data are collected in a uniform manner. For this reason, each variable and each response category within each variable has specifically been defined in a way that is designed to promote the collection and reporting of comparable minimal data.

Use of a standard format is essential for combining data from multiple investigators and locations. Various formats and coding schemes may be equally effective and could be used in individual studies or by agreement of the collaborating investigators. Suggestions for variable names and database structure are available at the website of the ISCoS (www.iscos.org.uk/international-sci-data-sets), and the National Institute of Neurological Disorders and Stroke (NINDS), the Common Data Elements Project website (www.CommonData Elements.ninds.nih.gov). ${ }^{4}$

\section{METHODS}

The first draft of the International SCI UTI Basic Data Set was made by a working group consisting of members approved by the American Spinal Injury Association (ASIA) and the ISCoS, together with a representative of the Executive Committee of the International Spinal Cord Injury Standards and

${ }^{1}$ Spinal Cord Injury and Disorders Unit, Hunter Holmes McGuire VA Medical Center, Physical Medicine and Rehabilitation, Virginia Commonwealth University, Richmond, VA, USA; ${ }^{2}$ University of Miami, Miami, FL, USA; ${ }^{3}$ McKay Urology, Carolinas Healthcare, Charlotte, NC, USA; ${ }^{4}$ Prince of Wales Spinal Unit, Prince of Wales Hospital, Sydney, New South Wales, Australia; ${ }^{5}$ Kessler Institute for Rehabilitation, West Orange, NJ, USA; ${ }^{6}$ Department of Clinical Microbiology, University of Copenhagen, Copenhagen, Denmark;

${ }^{7}$ Swiss Paraplegic Centre, Nottwil, Switzerland; ${ }^{8}$ University Hospital Antwerp, University Antwerp, Antwerp, Belgium and ${ }^{9}$ Clinic for Spinal Cord Injuries, Glostrup University Hospital and Faculty of Health Sciences, University of Copenhagen, Copenhagen, Denmark

Correspondence: Dr LL Goetz, Spinal Cord Injury and Disorders Unit, Hunter Holmes McGuire VA Medical Center, Physical Medicine and Rehabilitation, Virginia Commonwealth University, Richmond, VA 23249, USA

E-mail: lance.goetz@va.gov

Received 25 February 2013; revised 1 June 2013; accepted 3 June 2013; published online 30 July 2013 
Data Sets. The developmental process for the International SCI UTI Basic Data Set followed the steps given below:

(1) The working group of the International SCI UTI Basic Data Set finalized the first draft data set during a 3-day meeting in Copenhagen in November 2009. This was further elaborated by frequent e-mail contact among the group members including the development of a syllabus for the data set.

(2) The data set was reviewed by the members of the Executive Committee of the International SCI Standards and data sets.

(3) Comments from the committee members were discussed by the working group and appropriate changes were made to the data set.

(4) Members of the ISCoS Scientific Committee and ASIA Board were also asked to review the data set.

(5) Comments from the Committee/Board members were discussed in the working group, and a response was made and further adjustments of the data set were carried out.

(6) Relevant and interested scientific and professional (international) organizations and societies $(\sim 40)$ and individuals with an interest were also invited to review the data set. In addition, the data set was posted on the ISCoS and ASIA websites for 3 months to allow comments and suggestions.

(7) Comments were discussed by the working group and, where appropriate, minor adjustments to the data set were made.

(8) To conclude this part of the consultation, members of the ISCoS Scientific Committee, Executive Committee and ASIA Board received the data set for final review and approval.

(9) The International SCI UTI Basic Data Set was then further scrutinized by the team working on the NINDS, Common Data Element Project, in cooperation with the Executive Committee of the International SCI Standards and Data Set committees. ${ }^{4}$

(10) Endorsement of the data set will be obtained by the relevant (international) organizations and societies.

\section{RESULTS}

The complete data set form is included in the appendix. The complete data syllabus and data collection form are available at the ISCoS website (www.iscos.org.uk/international-sci-data-sets).

\section{Date of data collection}

This collection of data on UTI may be carried out at any time after the spinal cord lesion. Therefore, the date of data collection is imperative to be able to identify the data collected in relation to other data collected on the same individual at various time points.

\section{Length of time of onset of new symptom/symptoms}

This variable documents the new onset or increase in subjective $\operatorname{symptom}(\mathrm{s})$ that may present in individuals with spinal cord lesions who have a UTI. With respect to UTI, individuals with spinal cord lesions may have many signs and symptoms due to their spinal cord lesion or other problems. Therefore, it is important to determine that an individual's signs and symptoms are of a new onset or have increased and are not chronic in nature from a pre-existing or intercurrent problem.

An UTI is characterized by the new onset of $\operatorname{sign}(\mathrm{s}) / \operatorname{symptom}(\mathrm{s})$ (see below) accompanied by laboratory findings of a UTI, (bacteriuria, leukocyturia and positive urine culture) ${ }^{5}$ The individual would be expected to have an onset of symptoms within 2 weeks. However, it is possible that a person's UTI could develop into a chronic condition such as chronic testicular pain from epididymitis. It is important to note that individuals with a spinal cord lesion may have many other signs and symptoms in addition to the traditional signs and symptoms of a UTI in able-bodied individuals. Owing to alterations in sensation, some symptoms may be absent in individuals with spinal cord lesions. Other problems, such as autonomic dysreflexia, may develop or worsen due to a UTI.

\section{Signs and symptoms of UTI}

This variable documents the new onset or increase in signs and symptoms that may be experienced by individuals with spinal cord lesions who have a UTI. Symptoms are subjective reports given to the examiner. Signs are objective physical findings of the examiner. Individuals with spinal cord lesions may have many of the above signs and symptoms due to their spinal cord lesion or other problems. Many signs and symptoms do not constitute a justification for treatment. A UTI is characterized by the new onset of symptoms accompanied by laboratory findings (bacteriuria, leukocyturia and positive urine culture) of a $\mathrm{UTI}^{5}$ (National Institute on Disability and Rehabilitation Research criteria for UTI, 1992). For the purposes of the International SCI UTI Basic Data Set, only the signs and symptoms indicated above were chosen. Massa et al. ${ }^{6}$ found that cloudy urine had the highest accuracy $(83.1 \%)$, and leukocytes in the urine had the highest sensitivity $(82.8 \%)$ for the presence of UTI. Fever had very high specificity (99\%) but very low sensitivity (6.9\%). Autonomic dysreflexia data had low numbers and should be interpreted with caution. Kidney/bladder discomfort, increased spasticity, feeling sick, sense of unease, increased need to perform catheterization, feeling tired, incontinence and foul smelling urine all had high sensitivity $(77-95 \%)$ but very low specificity $(<50 \%)$.

The International SCI UTI Basic Data Set is not intended to include all possible symptoms. The 'other' field is available to document the symptoms outside of those listed.

Fever: elevated body temperature. In presence of a fever, one should check for signs of sepsis. Spinal cord-lesioned people, particularly those with cervical and high thoracic lesions, are prone to poikilothermia (inability to regulate core body temperature). Therefore, ambient temperature levels need to be taken into consideration while assessing fever. ${ }^{7}$ Additional research is necessary to better standardize temperature measurement and provide normative values for SCI. Thresholds for elevated body temperature (fever) and normal ranges for temperature variation in non-SCI adults are given in Table $1 .{ }^{8}$ The significant temperature ranges in children and infants have been previously published by El-Radhi. ${ }^{9}$ The potential clinical significance of lower temperature thresholds (than those given above) in children should be recognized.

Urinary incontinencelfailure of control or leaking around the catheter: the report of any involuntary leakage of urine. This may or may not be associated with urgency and need for increased catheterizations.

Spasticity: report on new or increased muscular hypertonicity compared with the patient's usual self-assessed baseline or on examination with increased resistance to stretch.

Malaise, lethargy or sense of unease: feeling tired or unwell, different from the person's usual state of health.

Cloudy urine: report that the urine is not clear. There may be report of mucus or sediment.

Table 1 Thresholds for elevated body temperature (fever) and normal ranges for temperature variation in non-spinal cord-injured adults ${ }^{8}$

Elevated body temperature

Observed normal rangesa

Oral at or above $38.2^{\circ} \mathrm{C}\left(101^{\circ} \mathrm{F}\right)$

$33.2-38.2^{\circ} \mathrm{C}\left(92-101^{\circ} \mathrm{F}\right)$

Axillary at or above $37^{\circ} \mathrm{C}\left(99^{\circ} \mathrm{F}\right)$

$35.5-37.0^{\circ} \mathrm{C}\left(96-99^{\circ} \mathrm{F}\right)$

Rectal at or above $37.8^{\circ} \mathrm{C}\left(100^{\circ} \mathrm{F}\right)$

$34.4-37.8{ }^{\circ} \mathrm{C}\left(94-100^{\circ} \mathrm{F}\right)$

Tympanic at or above $37.8^{\circ} \mathrm{C}\left(100^{\circ} \mathrm{F}\right)$

$35.4-37.8^{\circ} \mathrm{C}\left(96-100^{\circ} \mathrm{F}\right)$

Individuals with $\mathrm{SCl}$ who are prone to poikilothermia may need the above normal ranges to be adjusted for their normal temperature range within a given environmental temperature. The above temperature ranges are for non-SCl adults. Sund-Levander et al. ${ }^{8}$ also provide adult temperature values stratified by gender within their article. 
Malodorous urine: a distinct change in urine odor, with a strong foul smell that persists on change of catheter equipment.

Pyuria/leukocyturia: presence of white blood cells generated by the mucosal lining and observed on urinalysis.

Back pain: pain in the lower back below the rib cage-complaints of pain located on one or other side of the back just below the ribs (costovertebral angle region corresponding to the location of the kidney). Pain is elicited by palpation or percussion of the space over the kidneys. The costovertebral angle is formed by the lateral and downward curve of the lowest rib and the vertical column of the spine. This pain is frequently observed due to inflammation of a kidney.

Bladder pain: report of pain felt in the suprapubic or retropubic region (midline lower abdomen above the pubic symphysis), or with palpation. Usually increases with bladder filling and may persist after voiding.

Dysuria: pain and discomfort while voiding. Usually associated with localized inflammation but may be referred from pain in the bladder, prostate or sphincter.

Autonomic dysreflexia: in individuals with spinal cord lesions at T6 and above, patients complain of feelings related to a sudden onset of elevated blood pressure and other symptoms such as headache, sweating, flushing brought on by a noxious stimuli, such as bladder distention/bladder infection. ${ }^{10,11}$

\section{Urine dipstick test for nitrite and leukocyte esterase}

This variable documents the result of a urine dipstick test for nitrite and leukocyte esterase. Results for either may be positive, negative or unknown. An unknown result could be due to the test not being done or an unreadable/unusable result.

According to a meta-analysis of urine dipstick test accuracy, it was concluded that the dipstick test alone seems to be useful in all populations to exclude the presence of infection if the results of both nitrites and leukocyte esterase are negative. The usefulness of the dipstick test alone to rule in infection is uncertain. ${ }^{12}$ The only study with SCI individuals included in this analysis showed sensitivity of 0.79 , specificity of 0.99 , and positive and negative predictive values of 0.96 and 0.95 , respectively, when the combined nitrite and leukocyte esterase test was measured against culture. ${ }^{13}$ In a later study in an SCI population, there was found a positive predictive value at 1.00 to detect significant bacteriuria when both nitrites and leukocyte esterase tests were positive. ${ }^{14}$ When using the National Institute on Disability and Rehabilitation Research criteria for UTI, ${ }^{5}$ it was found that the reliance on dipstick testing could result in high rates of overtreatment and lower rates of undertreatment as compared with positive bacteriuria. ${ }^{14}$ It should also be considered that Enterococcus and some other bacteria are not able to reduce nitrates to nitrite and thus cannot be found with the dipstick test. Therefore, dipstick for nitrites and leukocyte esterase may be an initial indication, but should be followed by culture if there is an intention to treat a UTI in SCI individuals. One study compared the urine dipstick test and urine microscopic examination in SCI individuals and found these equally valuable. ${ }^{15}$

Microscopic analysis of urine, or urinalysis, is not a part of the routine clinical practice in many settings throughout the world. However, for sites where this is utilized, the microscopic analysis, particularly the bacteria and white blood cells, is very helpful in evaluating the presence of bacteriuria and quantitatively evaluating the degree of pyuria. ${ }^{16}$

\section{Culture of urine and sensitivity}

Culture of urine is one of the three major criteria in diagnosing UTIs, together with symptoms and immune response. ${ }^{6,17}$ Urine for culture should be collected as a clean-catch midstream technique from an immediately installed urine catheter or as a suprapubic aspiration from an installed catheter. ${ }^{17}$ It is not acceptable to collect a urine culture from a pre-existing catheter, from a collection bag or from a container. It is preferred that the sample is sent to the Clinical Microbiological Laboratory (CML) in a cool container to prevent growth of microorganisms. Alternatively, the freshly voided urine can be transferred to a 'dipslide' and then sent to the CML. UTIs in people with spinal cord lesions are, as per definition, complicated UTIs. ${ }^{18,19}$ All cultured microorganisms should be identified to the species level and quantified as colony-forming units per $\mathrm{ml}$ $\left(\mathrm{CFU} \mathrm{ml}{ }^{-1}\right) .{ }^{19}$ Any positive culture should be reported. This also includes information as to whether or not more than one microorganism is present. Persons with SCI commonly have more than one organism present in their urine culture. ${ }^{20} \mathrm{~A}$ value as low as $10 \mathrm{CFU} \mathrm{ml}^{-1}$ can, in some cases, be regarded as a significant finding. ${ }^{17,21}$ However, for the CML, $10^{3} \mathrm{CFU} \mathrm{ml}^{-1}$ is a more reliable finding with standardized inoculation with $10 \mu \mathrm{l}$ urine. ${ }^{22} \mathrm{~A}$ negative urine culture would indicate that there is no growth of any organism (bacteria or yeast). A positive culture could mean that there is growth of bacterial species, yeast species or both. Some CMLs may reject a specimen with more than two organisms as 'contaminated'. If there is certainty that the specimen has been collected correctly, it may be necessary to advocate with the CML for culture and speciation of all organisms.

In addition, an antibiogram providing a sensitivity pattern of relevant antimicrobials is mandatory. If the microorganism(s) cultured is/are resistant to three or more different antimicrobial agents to which the microorganism would normally be susceptible, it is defined as being multiresistant. ${ }^{23,24} \mathrm{~A}$ multiresistant microorganism, therefore, is not susceptible to more than three of the following eight antimicrobial agents: ampicillin/sulbactam, aztreonam, ceftazidime, ciprofloxacin, gentamicin, imipenem, piperacillin and trimethoprim/sulfamethoxazole. Colonization of multiresistant organisms in individuals with spinal cord lesions has been associated with neuropathic bladder, bladder management method (particularly indwelling catheterization), high rates of antibiotic use, mechanical ventilation and pressure ulcers. ${ }^{23,25-28}$ Precise identification is not only important to interpret the significance of the culture result, but also for interpretation of relapse or chronic/ biofilm infection. ${ }^{17,29}$ The antibiogram can also be used in revealing reinfection, relapse or chronic/biofilm infection. ${ }^{17}$ The latter can be supplemented with a measurement of specific antibody response to the pathogen. ${ }^{30}$ Finding of a chronic/biofilm infection can justify prolonged antibiotic treatment and may initiate examinations for urolithiasis, and focus on bladder-emptying techniques. ${ }^{19}$

\section{DISCUSSION}

The data in this International SCI UTI Basic Data Set shall be seen in conjunction with data in the International SCI Core Data Set, ${ }^{2}$ the International SCI Lower Urinary Tract Function Basic data Set, ${ }^{3}$ the International SCI Urodynamic Basic Data Set ${ }^{31}$ and the International Urinary Tract Imaging Basic Spinal Cord Injury Data Set. ${ }^{32}$ The International SCI Core Data Set includes, among other things, information on the date of birth and injury, gender, the cause of spinal cord lesion and neurological status, whereas the International SCI Lower Urinary Tract Function Basic Data Set includes variables on urinary tract impairment unrelated to spinal cord lesion, 
awareness of the need to empty the bladder, bladder emptying, bladder-emptying method, average number of voluntary bladder emptying per day during the last week, incontinence within the last 3 months, collecting appliances for urinary incontinence, any drugs for the urinary tract within the last year, surgical procedures on the urinary tract and any change in urinary symptoms within the last year. The International SCI Urodynamic Basic Data Set includes data on bladder sensation during filling cystometry, detrusor function, compliance during filing cystometry, function during voiding, detrusor leak-point pressure, maximum detrusor pressure, cystometric bladder capacity and post-void residual volume. The International Urinary Tract Imaging Basic Spinal Cord Injury Data Set includes data on urinary tract-imaging studies and findings.

This International SCI UTI Basic Data Set will need to be continually reviewed and, wherever necessary, updated. Ideas for improvement from individual or groups are welcome and will be addressed by the corresponding author. It should be recognized that the data set presented in this paper is a Basic Data Set and, as such, has been kept simple to provide condensed and easily assimilated information for follow-up consultations of people with spinal cord lesions. The International SCI UTI Basic Data Set is the fourth International SCI Urological Basic Data Set, and together with the International SCI Lower Urinary Tract Function Basic Data Set, the International SCI Urodynamic Basic Data Set and the International SCI Urinary Tract Imaging Basic Data Set, basic urological SCI information can be collected in individuals with spinal cord lesions admitted to SCI centers.

\section{CONCLUSION}

In summary, the essential components for the UTI Basic Data Set to be collected include the duration of signs or symptoms, reported signs and symptoms, urine dipstick results for nitrite and leukocyte esterase and urine culture.

\section{DATA ARCHIVING}

There were no data to deposit.

\section{CONFLICT OF INTEREST}

The authors declare no conflict of interest.

\section{ACKNOWLEDGEMENTS}

Coloplast A/S, Denmark has supported this study with this data set with an unconditional grant. We are thankful for comments and suggestions received from Susan Charlifue, Lawrence Vogel, Dan Lammertse, William Donovan, Inge Eriks Hoogland and Carly Rivers.

1 Biering-Sørensen F, Charlifue S, DeVivo M, Noonan V, Post M, Stripling T et al. International spinal cord injury data sets. Spinal Cord 2006; 44: 530-534.

2 DeVivo M, Biering-Sørensen F, Charlifue S, Noonan V, Post M, Stripling T et al. International Spinal Cord Injury Core Data Set. Spinal Cord 2006; 44: 535-540.

3 Biering-Sørensen F, Craggs M, Kennelly M, Schick E, Wyndaele JJ. International lower urinary tract function basic spinal cord injury data set. Spinal Cord 2008; 46 : 325-330.

4 Biering-Sørensen F, Charlifue S, Devivo MJ, Grinnon ST, Kleitman N, Lu Y et al. Incorporation of the International Spinal Cord Injury Data Set elements into the National Institute of Neurological Disorders and Stroke Common Data Elements. Spinal Cord 2010; 49: 60-64

5 National Institute on Disability and Rehabilitation Research Consensus Statement. The prevention and management of urinary tract infections amongst people with spinal cord injuries. J Am Paraplegia Soc 1992; 15: 194-204.
6 Massa LM, Hoffman JM, Cardenas DD. Validity, accuracy, and predictive value of urinary tract infection signs and symptoms in individuals with spinal cord injury on intermittent catheterization. J Spinal Cord Med 2009; 32: 568-573.

7 Guttmann L, Silver J, Wyndham CH. Thermoregulation in spinal man. J Physiol 1958; 142: 406-419.

8 Sund-Levander M, Forsberg C, Wahren LK. Normal oral, rectal, tympanic and axillary body temperature in adult men and women: a systematic literature review. Scand J Caring Sci 2002; 16: 122-128.

9 El-Radhi AS, Barry W. Thermometry in paediatric practice. Arch Dis Child 2006; 91 : 351-356.

10 Consortium for Spinal Cord Medicine. Acute Management of Autonomic Dysreflexia: Individuals with Spinal Cord Injury Presenting to Health-Care Facilities, 2nd edn. Paralyzed Veterans of America: Washington, D.C., USA, 2001, Available at http:// www.pva.org/site/c.ajIRK9NJLcJ2E/b.6305831/k.986B/ Guidelines_and_Publications.htm

11 Consortium for Spinal Cord Medicine. Bladder management for adults with spinal cord injury: a clinical practice guideline for health care providers. J Spinal Cord Med 2006; 29: 537-573.

12 Devillé WL, Yzermans JC, van Duijn NP, Bezemer PD, van der Windt DA, Bouter LM. The urine dipstick test useful to rule out infections. A meta-analysis of the accuracy. BMC Urol 2004; 4: 4: Review.

13 Tuel SM, Meythaler JM, Cross LL, McLaughlin S. Cost-effective screening by nursing staff for urinary tract infection in the spinal cord injured patient. Am J Phys Med Rehabil 1990; 69: 128-131.

14 Hoffman JM, Wadhwani R, Kelly E, Dixit B, Cardenas DD. Nitrite and leukocyte dipstick testing for urinary tract infection in individuals with spinal cord injury. J Spinal Cord Med 2004; 27: 128-132.

15 Faarvang KL, Müller P, Lomberg B, Biering-Sørensen F. Screening for bacteriuria in patients with spinal cord lesion: dipstick test, microscopic examination and urine culture. Spinal Cord 2000; 38: 106-108.

16 Baerheim A, Albrektsen G, Eriksen AG, Laerum E, Sandberg S. Quantification of pyuria by two methods: correlation and interobserver agreement. Scand J Prim Health Care 1989; 7: 83-86.

17 Stamm WE. Criteria for the diagnosis of urinary tract infection and for the assessment of therapeutic effectiveness. Infection 1992; 20 (Suppl 3), S151-S154.

18 Abrams P, Cardozo L, Fall M, Griffiths D, Rosier P, Ulmsten U et al. The standardization of terminology of lower urinary tract function: report from the standardisation sub-committee of the International Continence society. Neurourol Urodyn 2002; 21: $167-178$

19 Cardenas DD, Hooton TM. Urinary tract infection in persons with spinal cord injury. Arch Phys Med Rehabil 1995; 76: 272-280.

20 Linsenmeyer TA, Oakley A. Accuracy of individuals with spinal cord injury at predicting urinary tract infections based on their symptoms. J Spinal Cord Med 2003; 26: 352-357.

21 Warren JW, Abrutyn E, Hebel JR, Johnson JR, Schaeffer AJ, Stamm WE. Guidelines for antimicrobial treatment of uncomplicated acute bacterial cystitis and acute pyelonephritis in women. Infectious Diseases Society of America (IDSA). Clin Infect Dis 1999; 29: 745-758.

22 Frimodt-Møller N, Espersen F. Evaluation of calibrated 1 and 10 microL loops and dipslide as compared to pipettes for detection of low count bacteriuria in vitro. APMIS 2000; 108: 525-530.

23 Jung JY, Park MS, Kim SE, Park BH, Son JY, Kim EY et al. Risk factors for multi-drug resistant Acinetobacter baumannii bacteremia in patients with colonization in the intensive care unit. BMC Infect Dis 2010; 10: 228.

24 Moyo SJ, Aboud S, Kasubi M, Lyamuya EF, Maselle SY. Antimicrobial resistance among producers and non-producers of extended spectrum beta-lactamases in urinary isolates at a tertiary Hospital in Tanzania. BMC Res Notes 2010; 3: 348.

25 Girard R, Mazoyer MA, Plauchu MM, Rode G. High prevalence of nosocomial infections in rehabilitation units accounted for by urinary tract infections in patients with spinal cord injury. J Hospital Infect 2006; 62: 473-479.

26 Mylotte JM, Kahler L, Graham R, Young L, Goodnough S. Prospective surveillance for antibiotic-resistant organisms in patients with spinal cord injury admitted to an acute rehabilitation unit. Am J Infect Control 2000; 28: 291-297.

27 Thom JD, Wolfe V, Perkash I, Lin VW. Methicillin-resistant Staphylococcus aureus in patients with spinal cord injury. J Spinal Cord Med 1999; 22: 125-131.

28 Waites KB, Chen Y, DeVivo MJ, Canupp KC, Moser SA. Antimicrobial resistance in gram-negative bacteria isolated from the urinary tract in community-residing persons with spinal cord injury. Arch Phys Med Rehabil 2000; 81: 764-769.

29 Rosen DA, Hooton TM, Stamm WE, Humphrey PA, Hultgren SJ. Detection of intracellular bacterial communities in human urinary tract infection. PLoS Med 2007; 4: e329.

30 Moser C, Kriegbaum NJ, Larsen So, Høiby N, Biering-Sørensen F. Antibodies to urinary tract pathogens in patients with spinal cord lesions. Spinal Cord 1998; 36 : 613-616.

31 Biering-Sørensen F, Craggs M, Kennelly M, Schick E, Wyndaele JJ. International urodynamic basic spinal cord injury data set. Spinal Cord 2008; 46: 513-516.

32 Biering-Sørensen F, Craggs M, Kennelly M, Schick E, Wyndaele JJ. International urinary tract imaging basic spinal cord injury data set. Spinal Cord 2009; 47: $379-383$. 


\section{APPENDIX \\ URINARY TRACT INFECTION BASIC DATA SET (VERSION 1.0)}

Date of data collection: YYYYMMDD

Length of time of $\operatorname{sign}(\mathrm{s}) /$ symptom(s) (tick one only):
$\square$ Less than 1 day
$\square 1$ to 3 days
$\square 4$ days-1 week
$>1$ week-2 weeks

$\square>1$ month-3 months $\square>3$ months

$\square>2$ weeks-1 month

Signs/symptoms (tick all that apply):

$\square$ Fever

$\square$ Incontinence, onset or increase in episodes, including leaking around catheter

$\square$ Spasticity, increased

$\square$ Malaise, lethargy or sense of unease

$\square$ Cloudy urine (with or without mucus or sediment) with increased odor

$\square$ Pyuria

$\square$ Discomfort or pain over the kidney or bladder or during micturition

$\square$ Autonomic dysreflexia

$\square$ Other

Urine dipstick test for nitrite (tick one only):
$\square$ Negative
$\checkmark$ Positive
$\square$ Unknown

Urine dipstick test for leukocyte esterase (tick one only):

$\square$ Negative $\quad \square$ Positive $\square$ Unknown

Urine culture (tick one only):
$\square$ Negative
$\square$ Positive
Unknown

If positive, give species and amount of colony-forming units (CFU) $\mathrm{ml}^{-1}\left(10^{1}-10^{5} \mathrm{CFU} \mathrm{ml}{ }^{-1}\right)$, and the resistance pattern:

1) species, $\mathrm{CFU} \mathrm{ml}^{-1}$

Resistance pattern (tick one only): $\square$ Normal $\square$ Multidrug resistant (agents from 3 or more different drug classes)

2) species,

Resistance pattern (tick one only): $\square$ Normal $\square$ Multidrug resistant (agents from 3 or more different drug classes)

3) species, $\mathrm{CFU} \mathrm{ml}^{-1}$

Resistance pattern (tick one only): $\square$ Normal $\square$ Multidrug resistant (agents from 3 or more different drug classes)

4) species, $\mathrm{CFU} \mathrm{ml}^{-1}$

Resistance pattern (tick one only): $\square$ Normal $\square$ Multidrug resistant (agents from 3 or more different drug classes)

5) species, $\mathrm{CFU} \mathrm{ml}^{-1}$

Resistance pattern (tick one only): $\square$ Normal $\square$ Multidrug resistant (agents from 3 or more different drug classes) 